\title{
Dual-Reporter Drift Correction To Enhance the Performance of Electrochemical Aptamer-Based Sensors in Whole Blood
}

\author{
Hui Li, ${ }^{\dagger}$ Netzahualcóyotl Arroyo-Currás, ${ }^{\dagger}$ Di Kang, ${ }^{\dagger}$ Francesco Ricci, ${ }^{\S, \|}$ and Kevin W. Plaxco ${ }^{*}, \dagger,+$ \\ ${ }^{\dagger}$ Department of Chemistry and Biochemistry, University of California-Santa Barbara, Santa Barbara, California 93106, United States \\ ${ }^{\ddagger}$ Center for Bioengineering, University of California-Santa Barbara, Santa Barbara, California 93106, United States \\ ${ }^{\S}$ Department of Chemistry, University of Rome, Tor Vergata, Via della Ricerca Scientifica, 00133, Rome, Italy \\ "Consorzio Interuniversitario Biostrutture e Biosistemi "INBB”, Rome 00136, Italy
}

Supporting Information

\begin{abstract}
The continuous, real-time monitoring of specific molecular targets in unprocessed clinical samples would enable many transformative medical applications. Electrochemical aptamer-based (E-AB) sensors appear to be a promising approach to this end because of their selectivity (performance in complex samples, such as serum) and reversible, single-step operation. E-AB sensors suffer, however, from often-severe baseline drift when challenged in undiluted whole blood. In response we report here a dual-reporter approach to performing $\mathrm{E}-\mathrm{AB}$ baseline drift correction. The approach incorporates two redox reporters on the aptamer, one of which serves as the target-responsive sensor and the other, which reports at a distinct, nonoverlapping redox potential, serving as a driftcorrecting reference. Taking the difference in their relative signals largely eliminates the drift observed for these sensors in flowing, undiluted whole blood, reducing drift of up to $50 \%$ to less than $2 \%$ over many hours of continuous operation under these challenging conditions.
\end{abstract}

\footnotetext{
A major goal of bioanalytical chemistry has been the development of platforms supporting the rapid and convenient measurement of specific molecules directly in unprocessed clinical samples or even when deployed in situ in the body. ${ }^{1-5}$ Toward this goal we, ${ }^{6,7}$ and others, ${ }^{8}$ have previously described a broad class of electrochemical aptamer-based (E-AB) sensors for the detection of proteins, small molecules, and inorganic ions. ${ }^{9}$ E-AB sensors are comprised of an electrode-bound, redox reporter-modified aptamer that undergoes a binding-induced conformational change (Figure 1). This conformational change alters the positioning of the reporter relative to the electrode, producing a target-dependent change in current when the sensor is interrogated using square wave voltammetry. The reagentless, reversible nature of this signal transduction mechanism renders $\mathrm{E}-\mathrm{AB}$ sensors capable of supporting continuous monitoring. ${ }^{10}$ And because it mimics the conformation-linked signaling that occurs in natural chemoperception systems, ${ }^{11}$ this mechanism also renders $\mathrm{E}-\mathrm{AB}$ sensors among the most selective single-step biosensors reported to date, allowing for their multihour operation, for example, in undiluted blood serum. ${ }^{9,12,13}$ E-AB sensors suffer, however, from significant baseline drift when
}
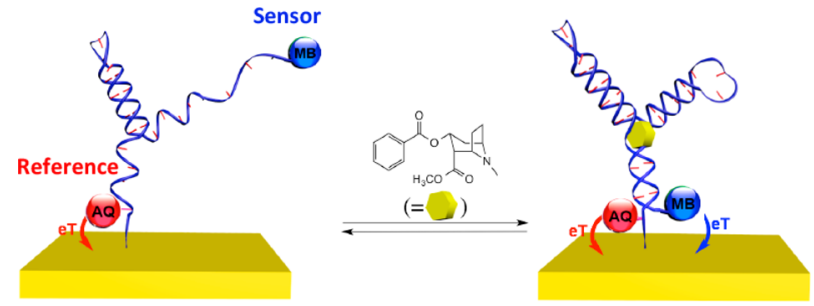

Figure 1. Dual-reporter drift correction for electrochemical aptamerbased sensors. Here we describe a dual-reporter approach to perform baseline drift correction for $\mathrm{E}-\mathrm{AB}$ sensors. To achieve this the aptamer is modified via the attachment of two redox reporters reporting at distinct, nonoverlapping potentials (here methylene blue, $\mathrm{MB}$, and anthraquinone, $A Q)$. The first, the sensing reporter, is placed at the distal end of the aptamer such that its output responds to any bindinginduced conformational changes. The second, the reference reporter, is placed at the electrode-proximal end of the aptamer such that its positioning relative to the electrode, and thus its output current is relatively insensitive to target binding, but it is equally sensitive to matrix effects responsible for signaling drift.

deployed directly in whole blood, likely due to the adherence of blood cells onto the electrode. ${ }^{14}$ In response, we demonstrate here a new solution to this baseline drift problem.

Our new approach to E-AB drift correction is analogous to a "dual-reporter" mechanism previously demonstrated for optical (fluorescent) sensors. ${ }^{15-22}$ In this a sensor's recognition "probe" is modified with two fluorophores reporting at distinct, nonoverlapping wavelengths. While both are responsive to environmental effects, instrumental artifacts, and variations in probe concentration, only one responds to the target. The other then serves as a reference that can be used to correct for these background effects, improving measurement accuracy and stability. Previously, the Ellington group has used an analogous approach to correct for sensor-to-sensor fabrication variability in electrochemical DNA-type sensors. ${ }^{23}$ Following this we describe here a dual-reporter approach to performing E-AB baseline-drift correction that enables continuous, real-time, long-duration measurements of specific molecules directly in flowing whole blood.

Our approach to drift correction relies on the use of two redox reporters conjugated to each aptamer probe (Figure 1), a

Received: August 18, 2016

Published: November 6, 2016 

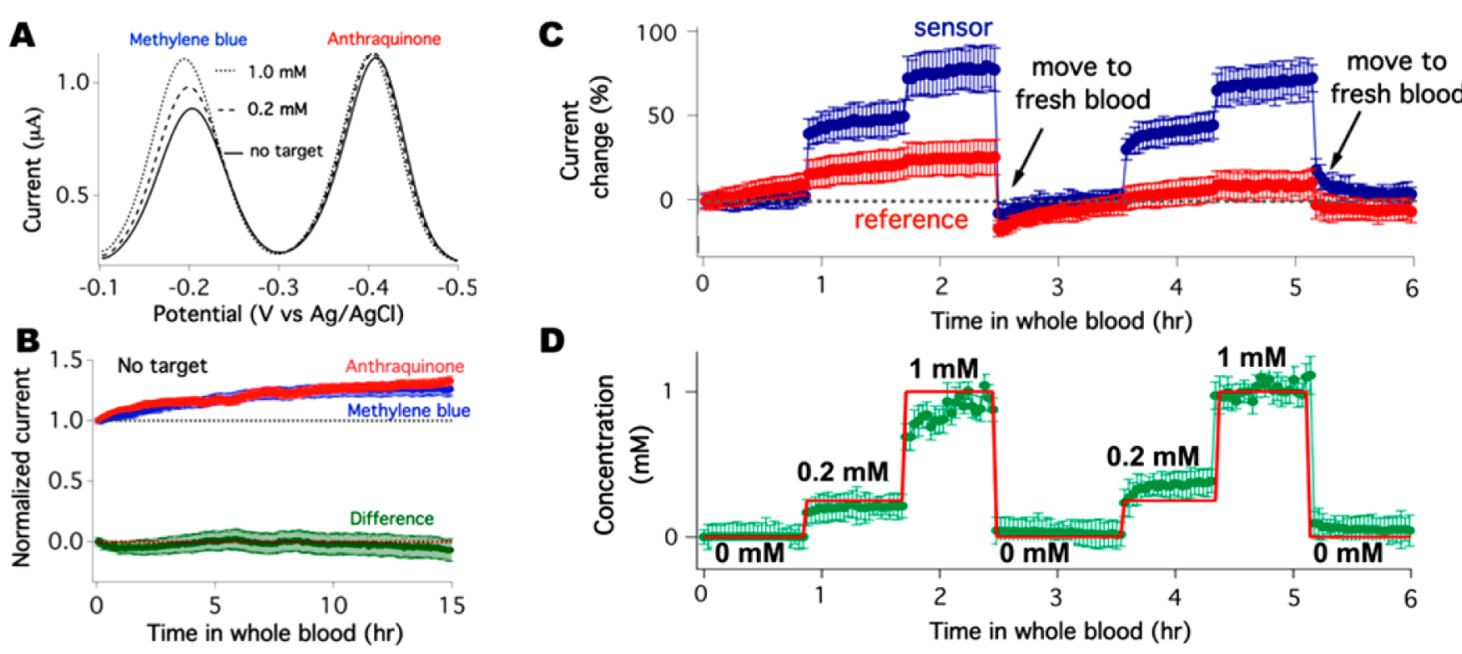

Figure 2. Dual-reporter drift correction for a cocaine-detecting E-AB sensor. (A) Voltammograms collected in phosphate buffered saline (PBS) demonstrate the extent to which, while the methylene blue (MB) reporter responds robustly to target, the anthraquinone (AQ) reporter is rather insensitive to the sensor's target. (B) Both redox reporters drift, however, when, as shown here, the sensor is placed in flowing, undiluted whole blood. But because they drift in concert, taking the difference between their normalized currents (green curve; eq 1) affords excellent drift correction. (C) The currents from the two reporters likewise drift in concert when the sensor is challenged with varying concentrations of its target in flowing whole blood. (D) After correction, the drift decreases significantly, recovering target concentration estimations (green curve) in close agreement with the actual concentrations of the drug spiked into the sample (red trace). The error bars, which reflect the standard deviation of measurements from multiple independently fabricated electrodes (Figures S2, S3), illustrate the magnitude of the sensor-to-sensor variation.

sensing reporter, here methylene blue $(\mathrm{MB})$, and a reference reporter, here anthraquinone (AQ). We selected these reporters for three reasons. First, their potentials do not overlap, allowing for the simultaneous monitoring of both. Second, both are reasonably stable. ${ }^{24}$ And, finally, their physical properties are sufficiently similar that they respond in concert to the environmental changes that cause drift. To perform drift correction we placed the sensing reporter (i.e., MB) on the distal end (away from the electrode) of the aptamer. In this position the efficiency with which it approaches the electrode to transfer electrons depends sensitively on the (binding-defined) conformation of the aptamer. We placed the reference reporter (i.e., AQ), in contrast, on the nucleobase proximal to the electrode, rendering its distance from the electrode largely independent of the aptamer's conformation. Thus, while both reporters respond to the environmental effects that cause drift, only the sensing reporter also responds to the target.

Motivated by the argument that monitoring drug levels is a particularly important application of in-blood measurements we used a sensor employing the cocaine-binding aptamer of Stojanovic $^{25,26}$ as our first test bed. We modified the distal end of the aptamer with $\mathrm{MB}$ and its proximal end with AQ. Using square wave voltammetry to interrogate the resultant sensor in phosphate-buffered saline (PBS) we observed redox peaks at $-0.26 \mathrm{~V}$ and $-0.42 \mathrm{~V}$, corresponding to the reduction of $\mathrm{MB}$ and $\mathrm{AQ}$ respectively (Figure $2 \mathrm{~A}$ ). As expected, the current originating from the $\mathrm{MB}$ reporter increases significantly and monotonically upon spiking with increasing concentrations of cocaine. The current from the AQ reporter, in contrast, is largely insensitive to the target, suggesting that it might prove a suitable drift-correction reference.

To test the ability of the AQ reporter to provide stable drift correction we first challenged a set of these cocaine-detecting sensors in flowing, undiluted whole blood using an in vitro system meant to mimic circulation in the vasculature (Figure S1). Over the course of $15 \mathrm{~h}$ in flowing whole blood the currents arising from both $\mathrm{MB}$ and $\mathrm{AQ}$ drift by $25 \%-30 \%$
(Figure 2B). The two currents, however, drift in concert, as required for dual-reporter drift correction. To perform such a correction, we calculate the change in relative currents from

$$
S_{\text {cor }}=\frac{i_{\mathrm{MB}}}{i_{\mathrm{MB} 0}}-\frac{i_{\mathrm{AQ}}}{i_{\mathrm{AQ} 0}}
$$

in which $S_{\text {cor }}$ is the corrected relative signal change, $i_{\mathrm{MB}}$ and $i_{\mathrm{AQ}}$ are the peak currents from the $\mathrm{MB}$ and $\mathrm{AQ}$ respectively, and $i_{\mathrm{MB} 0}$ and $i_{\mathrm{AQ} 0}$ are the peak currents collected in the absence of target at the first data point in each experiment. In sharp contrast to the raw $\mathrm{MB}$ and AQ currents, the drift in $S_{\text {cor }}$ is less than $2 \%$ after $15 \mathrm{~h}$ (green trace, Figure $2 \mathrm{~B}$ ). And while the drift in raw current observed for individual sensors often varies dramatically (presumably due to the stochastic formation of macroscopic aggregates of blood cells on the sensor surface), the drift correction is quite reproducible (Figure S2).

Dual-reporter baseline correction also corrects for sensor drift when the sensor is being challenged with its specific molecular target. To demonstrate this we tested dual-reporter cocaine sensors against varying pulses of the drug in flowing whole blood over the course of several hours. In both the presence and absence of target we again observe significant ( $\sim 10 \%$ per hour), continuous drift in both the $\mathrm{MB}$ and $\mathrm{AQ}$ currents (Figure 2C). In contrast, $S_{\text {cor }}$ and, from that, the predicted concentration drift far less (Figure 2D).

To determine whether dual-reporter drift correction is general, we next characterized $\mathrm{E}-\mathrm{AB}$ sensors employing dualmodified aptamers binding the aminoglycoside antibiotics ${ }^{27}$ and the cancer chemotherapeutic doxorubicin. ${ }^{14}$ In contrast to the cocaine-binding aptamer, which adopts a trefoil fold, ${ }^{27}$ these aptamers fold into a hairpin structure, ${ }^{28,14}$ thus providing further insights into the generality (aptamer-independence) of our approach. Once again we modified each with an MB on its distal end and an AQ on its proximal end.

The dual-reporter approach effectively corrects baseline drift for both aminoglycoside- and doxorubicin-detecting sensors. Upon challenging the former in buffer with kanamycin, for 

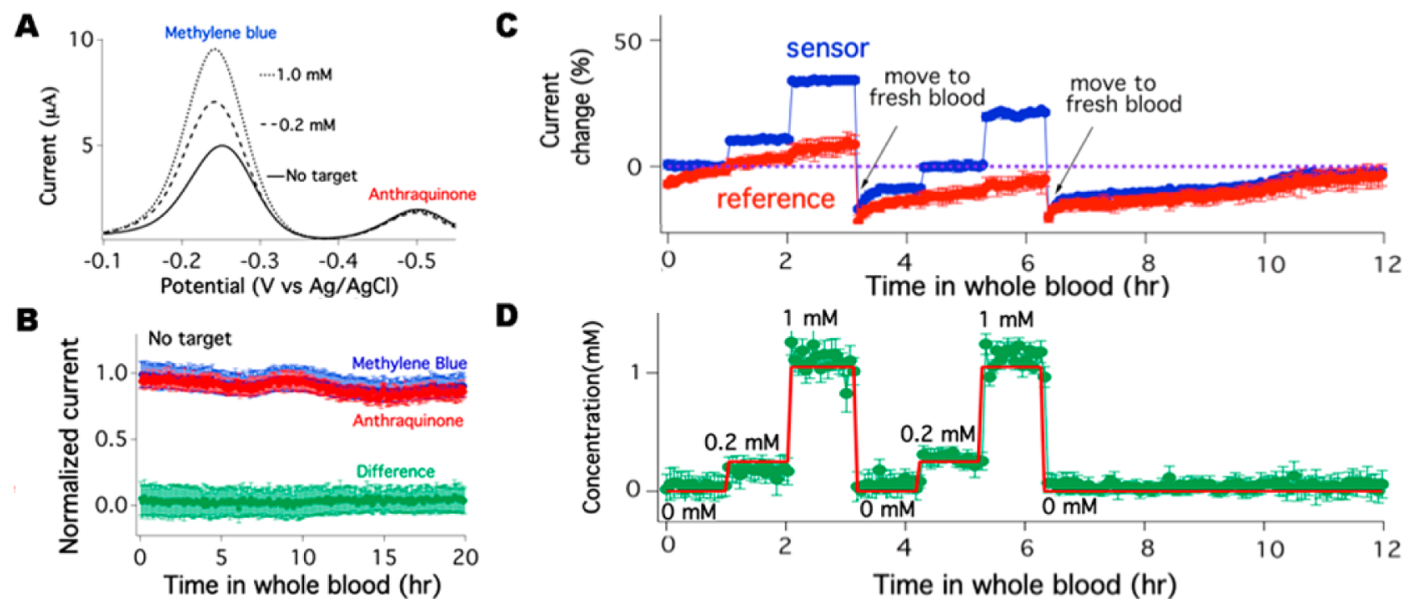

Figure 3. Dual-reporter drift correction for a kanamycin-detecting E-AB sensor. (A) Voltammograms collected in PBS buffer demonstrate the extent to which, while the methylene blue $(\mathrm{MB})$ reporter responds robustly to the sensor's target, the anthraquinone (AQ) reporter is rather insensitive. (B) Both redox reporters drift, however, when the sensor is placed in flowing, undiluted whole blood. But because they drift in concert, taking the difference between their normalized currents (green curve; eq 1) once again affords excellent drift correction. (C) The currents from the two reporters drift in concert when the sensor is spiked with varying concentrations of the target in flowing whole blood. (D) After correction, the drift decreases significantly, recovering target concentration estimations (green curve) in close agreement with the actual concentrations of the drug spiked into the sample (red trace). The error bars, which reflect the standard deviation of measurements from multiple independently fabricated electrodes (Figures S5, S6), illustrate the magnitude of the sensor-to-sensor variation.

example, we find that the current from the $\mathrm{MB}$ reporter increases significantly while that from the AQ reporter increases by less than $5 \%$ (Figure $3 \mathrm{~A}$ ). Upon challenging it in flowing whole blood for $20 \mathrm{~h}$ the currents from both reporters drift by $15 \%-20 \%$, which is reduced to less than $2 \%$ upon correction (Figure $3 \mathrm{~B}$ ). We then tested the sensor against varying pulses of kanamycin in flowing whole blood, finding that both currents drift by $\sim 7 \%$ per hour and also exhibit discontinuous "jumps" of order $25 \%$ when removed from drug-containing whole blood and returned to a drug-free sample (Figure 3C). Again, however, dual-reporter drift correction reduces the drift in estimated drug concentration to less than $2 \%$ per hour and effectively eliminates the jumps seen upon transfer (Figure 3D). The dual-reporter approach also effectively corrects baseline drift for the doxorubicin sensor, reducing a drift of more than $50 \%$ over $8 \mathrm{~h}$ to less than 5\% (Figure S8). Unfortunately, however, doxorubicin is redox active at the same potential as $\mathrm{AQ}$ and thus we were precluded from studying the performance of this sensor in the presence of its target.

Here we have demonstrated a dual-reporter approach to performing baseline drift correction for $\mathrm{E}-\mathrm{AB}$ sensors. The approach largely eliminates the drift seen when $\mathrm{E}-\mathrm{AB}$ sensors are deployed directly in flowing, undiluted whole blood, reducing drift in the raw electrochemical signals of up to $50 \%$ to less than 5\% over many hours. Moreover, the approach corrects both the steady drift seen in flowing blood and the dramatic jumps in signal seen when the sensor is physically moved into a fresh sample. Given these results we are optimistic that, by analogy to the optical counterpart that inspired it, dual-reporter drift correction will be applicable to a wide variety of electrochemical sensor architectures.

\section{ASSOCIATED CONTENT}

\section{S Supporting Information}

The Supporting Information is available free of charge on the ACS Publications website at DOI: 10.1021/jacs.6b08671.

Detailed description of the experimental procedures, figures, and references (PDF)

\section{AUTHOR INFORMATION}

\section{Corresponding Author}

*kwp@chem.ucsb.edu

\section{ORCID}

Netzahualcóyotl Arroyo-Currás: 0000-0002-2740-6276

\section{Notes}

The authors declare no competing financial interest.

\section{ACKNOWLEDGMENTS}

The biosensor work at UCSB was carried out at the Institute for Collaborative Biotechnologies (supported by the Army Research Office, Grant W911NF-09-0001) and with partial support from the W. M. Keck Foundation. Dr. Li was partially supported by the Swiss National Science Foundation with an "Early Postdoc Mobility fellowship". Dr. Li also appreciates helpful discussions with Dr. Shaoguang Li, Dr. Philippe Dauphin Ducharme, and Dr. Martin Kurnik.

\section{REFERENCES}

(1) Chen, Q.; Andersson, A.; Mecklenburg, M.; Xie, B. Clin. Microbiol. Infect. 2013, 19, 869.

(2) Stewart, A. J.; Hendry, J.; Dennany, L. Anal. Chem. 2015, 87, 11847.

(3) Wilson, G. S.; Gifford, R. Biosens. Bioelectron. 2005, 20, 2388

(4) Ward, W. K.; House, J. L.; Birck, J.; Anderson, E. M.; Jansen, L. B. Diabetes Technol. Ther. 2004, 6, 389.

(5) Rodbard, D. Diabetes Technol. Ther. 2016, 18 (Suppl 2), S2.

(6) Baker, B. R.; Lai, R. Y.; Wood, M. S.; Doctor, E. H.; Heeger, A. J.; Plaxco, K. W. J. Am. Chem. Soc. 2006, 128, 3138.

(7) Cash, K. J.; Ricci, F.; Plaxco, K. W. J. Am. Chem. Soc. 2009, 131, 6955.

(8) Zuo, X.; Song, S.; Zhang, J.; Pan, D.; Wang, L.; Fan, C. J. Am. Chem. Soc. 2007, 129, 1042.

(9) Lubin, A. A.; Plaxco, K. W. Acc. Chem. Res. 2010, 43, 496.

(10) Xiao, Y.; Lai, R. Y.; Plaxco, K. W. Nat. Protoc. 2007, 2, 2875.

(11) Plaxco, K. W.; Soh, H. T. Trends Biotechnol. 2011, 29, 1.

(12) Lubin, A. A.; Lai, R. Y.; Baker, B. R.; Heeger, A. J.; Plaxco, K. W. Anal. Chem. 2006, 78, 5671. 
(13) Valleé-Belisle, A.; Ricci, F.; Uzawa, T.; Xia, F.; Plaxco, K. W. J. Am. Chem. Soc. 2012, 134, 15197.

(14) Ferguson, B. S.; Hoggarth, D. A.; Maliniak, D.; Ploense, K.; White, R. J.; Woodward, N.; Hsieh, K.; Bonham, A. J.; Eisenstein, M.; Kippin, T. E.; Plaxco, K. W.; Soh, H. T. Sci. Transl. Med. 2013, 5, 213.

(15) Lu, H.; Jin, Y.; Tian, Y.; Zhang, W.; Holl, M. R.; Meldrum, D. R. J. Mater. Chem. 2011, 21, 19293.

(16) Wu, S.; Li, Z.; Ha, J.; Han, S. Chem. Commun. 2011, 47, 11276.

(17) Huang, J.; Ying, L.; Yang, X.; Yang, Y.; Quan, K.; Wang, H.; Xie, N.; Ou, M.; Zhou, Q.; Wang, K. Anal. Chem. 2015, 87, 8724.

(18) Chen, A. K.; Davydenko, O.; Behlke, M. A.; Tsourkas, A. Nucleic Acids Res. 2010, 38, 148.

(19) Chen, C.; Xiang, X.; Liu, Y.; Zhou, G.; Ji, X.; He, Z. Biosens. Bioelectron. 2014, 58, 205.

(20) Xiang, D.-S.; Zhou, G.-H.; Luo, M.; Ji, X.-H.; He, Z.-K. Analyst 2012, 137, 3787.

(21) Coskun, A. F.; Cetin, A. E.; Galarreta, B. C.; Alvarez, D. A.; Altug, H.; Ozcan, A. Sci. Rep. 2014, 4, 6789.

(22) Karlstrom, A.; Nygren, P. Anal. Biochem. 2001, 295, 22.

(23) Du, Y.; Lim, B. J.; Li, B.; Jiang, Y. S.; Sessler, J. L.; Ellington, A. D. Anal. Chem. 2014, 86, 8010.

(24) Kang, D.; Ricci, F.; White, R. J.; Plaxco, K. W. Anal. Chem. 2016, $88,10452$.

(25) Stojanovic, M. N.; de Prada, P. D.; Landry, D. W. J. Am. Chem. Soc. 2001, 123, 4928.

(26) Swensen, J. S.; Xiao, Y.; Ferguson, B. S.; Lai, R. Y.; Heeger, A. J.; Plaxco, K. W.; Soh, T. J. Am. Chem. Soc. 2009, 131, 4262.

(27) Rowe, A. A.; Miller, E. A.; Plaxco, K. W. Anal. Chem. 2010, 82, 7090.

(28) Jiang, L.; Patel, D. Nat. Struct. Biol. 1998, 5, 769. 